\title{
A REMARK ON THE UNIQUENESS OF WEIGHTED MARKOV BRANCHING PROCESSES
}

\author{
ANYUE CHEN, ${ }^{*}$ The University of Hong Kong and The University of Greenwich \\ PHIL POLLETT, ${ }^{* * * * *}$ The University of Queensland \\ JUNPING LI, ${ }^{* * * *}$ Central South University, Changsha \\ HANJUN ZHANG, ${ }^{* * * * * * *}$ The University of Queensland
}

\begin{abstract}
We present an elegant uniqueness criterion for the weighted Markov branching process in the potentially explosive case.
\end{abstract}

Keywords: Markov branching process; regularity; uniqueness

2000 Mathematics Subject Classification: Primary 60J27

Secondary $60 \mathrm{~J} 80$

\section{Introduction}

The basic property that governs the evolution of Markov branching processes (MBPs) is the branching property, i.e. that particles behave independently producing descendants according to the same rule. This greatly simplifies their analysis; their study has proved to be a very fruitful area of research in stochastic processes (standard references, among others, are Harris (1963), Athreya and Ney (1972), and Asmussen and Hering (1983)). However, since particles may interact, through collision or some other mechanism, the branching property may be lost. For this reason, more general branching models have been proposed (see, for example, Athreya and Jagers (1997)). A particularly interesting class, which we call here weighted Markov branching processes (WMBPs), was considered by R. R. Chen (1997). Using the methods and techniques developed by M. F. Chen (1992), R. R. Chen addressed questions concerning regularity and eventual extinction (see also A. Y. Chen (2002a), (2002b)), but left open the question of uniqueness in the potentially explosive case (see Definition 1, below). Our aim here is to establish a uniqueness criterion which is easy to check. We begin with some definitions.

Definition 1. A $q$-matrix $Q=\left(q_{i j}, i, j \in \mathbb{Z}_{+}\right)$is called a weighted branching $q$-matrix (WB-q-matrix) if

$$
q_{i j}= \begin{cases}w_{i} b_{j-i+1}, & i \geq 1, j \geq i-1, \\ 0, & \text { otherwise }\end{cases}
$$

\footnotetext{
Received 22 June 2005.

* Postal address: Department of Statistics and Actuarial Science, The University of Hong Kong, Pokfulam Road, Hong Kong. Email address: achen@hkucc.hku.hk

** Postal address: Department of Mathematics, The University of Queensland, Brisbane, QLD 4072, Australia.

*** Email address: pkp@maths.uq.edu.au

**** Postal address: School of Mathematical Sciences and Computing Technology, Central South University, Changsha, 410075, P. R. China. Email address: jpli@mail.csu.edu.cn

******Email address: hjz@maths.uq.edu.au
} 
where $w_{j}>0(j \geq 1), b_{0}>0, b_{j} \geq 0(j \geq 2)$, and $-b_{1}=\sum_{j \neq 1}^{\infty} b_{j}$. It is called potentially explosive if $\sum_{k=1}^{\infty} 1 / w_{k}<\infty$ and subexplosive if $\sum_{k=1}^{\infty} 1 / w_{k}=\infty$.

In order that the branching property holds for the ordinary MBP it is necessary that its transition function obeys the Kolmogorov forward equation. Guided by this fact, we use the following definition.

Definition 2. A $Q$-process is called a weighted Markov branching process (WMBP) if its transition function $\boldsymbol{P}(t)=\left(p_{i j}(t), i, j \in \mathbb{Z}_{+}, t>0\right)$ satisfies the Kolmogorov forward equation $\boldsymbol{P}^{\prime}(t)=\boldsymbol{P}(t) \boldsymbol{Q}$.

Chen et al. (2005) derived regularity conditions for WMBPs in terms of $d:=b_{0}$ (death rate) and $m_{b}:=\sum_{j=1}^{\infty} j b_{j+1}$ (net birth rate). They proved that the subexplosive WB- $q$-matrix is almost regular (meaning that it is regular if $m_{b}$ is finite), and they obtained a simple criterion for regularity in the potentially explosive case.

Proposition 1. The potentially explosive WB-q-matrix $Q$ is regular if and only if $d \geq m_{b}$.

From Proposition 1 we can see that a potentially explosive $\boldsymbol{Q}$ is explosive whenever $d<$ $m_{b} \leq \infty$, and general theory then dictates that there are infinitely many $\boldsymbol{Q}$-processes, including infinitely many honest ones (see Anderson (1991) or Yang (1990)). However, for uniqueness of the WMBP, we require the forward equation to hold. The following uniqueness criterion was obtained by Chen et al. (2005).

Proposition 2. If $\boldsymbol{Q}$ is potentially explosive and $d<m_{b} \leq \infty$, then there is only one WMBP if and only if $\sum_{n=1}^{\infty} R_{n}=\infty$, where

$$
\begin{aligned}
& R_{0}=1 \\
& R_{n}=\frac{1}{b_{0} w_{n+1}}\left(1+\sum_{k=1}^{n} w_{k} \tau_{n-k+2} R_{k-1}\right) \text { for } n \geq 1,
\end{aligned}
$$

with $\tau_{n}=\sum_{j=n}^{\infty} b_{j}, n \geq 2$.

However, this criterion is not easy to check in all cases. We shall provide an alternative criterion, which is much easier to verify.

\section{The main result}

Let $U$ be the generating function defined by $U(s)=\sum_{j=0}^{\infty} b_{j} s^{j}$. It is well known that if $d<m_{b} \leq \infty$ then $U(s)=0$ has two distinct roots, $q$ and 1 , with $0<q<1$; we shall always denote the smaller of these by $q$.

Theorem 1. If the WB-q-matrix $Q$ is potentially explosive and $d<m_{b} \leq \infty$, then there is more than one WMBP if and only if

$$
\sum_{n=1}^{\infty} \frac{1}{w_{n} q^{n}}<\infty .
$$

Moreover, if (2) holds then there are infinitely many WMBPs, exactly one of which is honest (it is not the minimal $Q$-process). If (2) fails to hold then there is only one WMBP, being the (dishonest) minimal $\mathbf{Q}$-process. 
The following immediate corollary improves on the conclusions of Chen et al. (2005).

Corollary 1. Suppose that $\boldsymbol{Q}$ is potentially explosive and $d<m_{b} \leq \infty$. If

$$
\liminf _{n \rightarrow \infty} \sqrt[n]{w_{n}}<\frac{1}{q}
$$

then there is only one WMBP, being the (dishonest) minimal $Q$-process; while if

$$
\liminf _{n \rightarrow \infty} \sqrt[n]{w_{n}}>\frac{1}{q}
$$

then there is only one honest WMBP (which is not the minimal $Q$-process), together with infinitely many dishonest WMBPs.

In order to prove Theorem 1, we will need two technical lemmas. Define $G$ by

$$
G(s)=\frac{q-s}{U(s)} .
$$

Lemma 1. Viewed as a complex function, $G(z)$ is analytic, at least on the unit disk $\mathscr{D}=$ $\{z,|z|<1\}$, and has a Taylor expansion

$$
G(z)=\sum_{n=0}^{\infty} g_{n} z^{n}
$$

whose coefficients satisfy

$$
0<g_{n} \leq g_{0}=\frac{q}{d}, \quad n \geq 0 .
$$

Proof. Since $d<m_{b} \leq \infty$, we may let $\rho_{0}=d / q\left(=b_{0} / q\right)$ and $\rho_{k}=\sum_{m=1}^{\infty} b_{k+m} q^{m-1}$, $k \geq 1$. It is easy to see that $\left(\rho_{k}, k \geq 0\right)$ is a nonnegative sequence with $\rho_{0}>0$ and $\sum_{k=1}^{\infty} \rho_{k}=$ $\rho_{0}$. A little algebra together with (3) then immediately yields

$$
G(z)=\left(\rho_{0}-\sum_{k=1}^{\infty} \rho_{k} z^{k}\right)^{-1} .
$$

Since $\rho_{0}-\sum_{k=1}^{\infty} \rho_{k} z^{k}$ is analytic on $\mathscr{D}$, and $\left|\rho_{0}-\sum_{k=1}^{\infty} \rho_{k} z^{k}\right| \geq \rho_{0}(1-|z|)>0$ for all $z \in \mathscr{D}$, we deduce that $G$ is analytic on $\mathscr{D}$ and, so, has the Taylor expansion (4).

Since (4) and (6) hold for all $z \in \mathscr{D}$, we obtain $\rho_{0} g_{0}=1$ and $\rho_{0} g_{n}=\sum_{k=1}^{n} \rho_{k} g_{n-k}, n \geq 1$, and then (5) follows immediately.

Lemma 2. Define $\left(T_{n}, n \geq 0\right)$ by $T_{0}=w_{1}$ and $T_{n}=w_{n+1} R_{n}, n \geq 1$, where $R_{n}$ is given by (1). Then $T(s):=\sum_{n=0}^{\infty} T_{n} s^{n}$ has radius of convergence $q$ and

$$
T(s) U(s)=s+d w_{1}(1-s), \quad 0 \leq s<q .
$$

Proof. Theorem 5 of Chen et al. (2005) established that the power series $T(s)$ has radius of convergence $q$. Now we rewrite (1) as $T_{n}=\left(1+\sum_{k=1}^{n} T_{k-1} \tau_{n-k+2}\right) / b_{0}$. A little algebra then establishes (7).

We are now ready to prove Theorem 1 . 
Proof of Theorem 1. By (3) and (4) we have, for $|s|<q$,

$$
\frac{1}{U(s)}=\frac{G(s)}{q-s}=\frac{1}{q} \sum_{m=0}^{\infty}\left(\frac{s}{q}\right)^{m} \sum_{n=0}^{\infty} g_{n} s^{n}=\frac{1}{q} \sum_{n=0}^{\infty} C_{n} s^{n},
$$

where

$$
C_{n}=\sum_{k=0}^{n} g_{k}\left(\frac{1}{q}\right)^{n-k} \equiv \sum_{k=0}^{n}\left(\frac{1}{q}\right)^{k} g_{n-k} .
$$

Now, (8) together with (7) yields, for $|s|<q$,

$$
T(s)=\frac{d w_{1}}{q} \sum_{n=0}^{\infty} C_{n}(1-s) s^{n}+\frac{1}{q} \sum_{n=0}^{\infty} C_{n} s^{n+1} .
$$

But, $T(s)=\sum_{n=0}^{\infty} T_{n} s^{n}=\sum_{n=0}^{\infty} w_{n+1} R_{n} s^{n}$ and, since (9) holds for all $|s|<q$, we find that

$$
R_{n}=\frac{C_{n}-C_{n-1}}{w_{n+1}} \frac{d w_{1}}{q}+\frac{C_{n-1}}{w_{n+1}} \frac{1}{q}, \quad n \geq 1 .
$$

The sequence $\left(C_{n}, n \geq 0\right)$ is nonnegative and increasing because

$$
C_{n}-C_{n-1}=g_{n}+\frac{1-q}{q} \sum_{k=1}^{n}\left(\frac{1}{q}\right)^{k-1} g_{n-k}>0 .
$$

Therefore, both terms on the right-hand side of (10) are nonnegative. Thus, by Proposition 2, the WMBP is not unique if and only if both $\sum_{n=1}^{\infty}\left(C_{n}-C_{n-1}\right) / w_{n+1}<\infty$ and $\sum_{n=1}^{\infty} C_{n-1} / w_{n+1}<\infty$, or, equivalently,

$$
\sum_{n=1}^{\infty} \frac{C_{n}}{w_{n+1}}<\infty
$$

The proof will be complete if we can show that this is equivalent to (2). First assume that (2) holds. Since $\left(g_{n}, n \geq 0\right)$ is bounded above by $q / d$ (see (5)), we have

$$
C_{n} \leq \frac{q}{d} \sum_{k=0}^{n}\left(\frac{1}{q}\right)^{k}=\frac{q^{2}}{d(1-q)}\left(\left(\frac{1}{q}\right)^{n+1}-1\right) \leq \frac{q^{2}}{d(1-q)}\left(\frac{1}{q}\right)^{n+1}
$$

for all $n \geq 0$. Therefore, by (2),

$$
\sum_{n=1}^{\infty} \frac{C_{n}}{w_{n+1}} \leq \frac{q^{2}}{d(1-q)} \sum_{n=2}^{\infty} \frac{1}{w_{n} q^{n}}<\infty
$$

thus establishing (11). Conversely, if (11) holds then, since $C_{n} \geq g_{0}(1 / q)^{n}$ for all $n \geq 0$, we have

$$
\sum_{n=1}^{\infty} \frac{g_{0}}{w_{n+1}}\left(\frac{1}{q}\right)^{n}<\infty
$$

and so (2) follows because $0<g_{0}<\infty$ and $q>0$. This completes the proof. 


\section{Acknowledgements}

The work of Anyue Chen was substantially supported by a grant from the Research Grants Council of the Hong Kong Special Administrative Region, China, Project HKU 7010/06P. The work of Phil Pollett and Hanjun Zhang was supported by the Australian Research Council Centre of Excellence for Mathematics and Statistics of Complex Systems.

\section{References}

Anderson, W. J. (1991). Continuous-Time Markov Chains: An Applications-Oriented Approach. Springer, New York. Asmussen, S. And Hering, H. (1983). Branching Processes. Birkhäuser, Boston, MA.

Athreya, K. B. And Jagers, P. (1997). Classical and Modern Branching Processes. Springer, Berlin.

Athreya, K. B. And Ney, P. E. (1972). Branching Processes. Springer, Berlin.

Chen, A., Pollett, P., Zhang, H. and Cairns, B. (2005). Uniqueness criteria for continuous-time Markov chains with general transition structure. Adv. Appl. Prob. 37, 1056-1074.

Chen, A. Y. (2002a). Ergodicity and stability of generalized Markov branching processes with resurrection. J. Appl. Prob. 39, 786-803.

Chen, A. Y. (2002b). Uniqueness and extinction properties of generalized Markov branching processes. J. Math. Anal. Appl. 274, 482-494.

Chen, M. F. (1992). From Markov Chains to Non-Equilibrium Particle Systems. World Scientific, Singapore.

Chen, R. R. (1997). An extended class of time-continuous branching processes. J. Appl. Prob. 34, 14-23.

Harris, T. H. (1963). The Theory of Branching Processes. Springer, Berlin.

YAnG, X. Q. (1990). The Construction Theory of Denumerable Markov Processes. John Wiley, Chichester. 